\title{
On optimal strategies for utility maximizers in the Arbitrage Pricing Model
}

\author{
Miklós Rásonyi*
}

July 18, 2016

\begin{abstract}
We consider a popular model of microeconomics with countably many assets: the Arbitrage Pricing Model. We study the problem of optimal investment under an expected utility criterion and look for conditions ensuring the existence of optimal strategies. Previous results required a certain restrictive hypothesis on the tails of asset return distributions. Using a different method, we manage to remove this hypothesis, at the price of stronger assumptions on the moments of asset returns.
\end{abstract}

MSC 2010 subject classification: Primary: 91B16, 91B25; secondary: 49M99, 93E20

Keywords: utility maximization, large financial markets, optimal strategies, risk-neutral measures

\section{Introduction}

The Arbitrage Pricing Model was first proposed in Ross (1976). The related mathematics was clarified by Huberman (1982). This model has become standard material in courses of economic theory, Huang \& Litzenberger (1988), and also inspired far-reaching developments in financial mathematics, the theory of so-called "large financial markets": see e.g. Kabanov \& Kramkov (1994,1998), Klein \& Schachermayer (1996a,1996b), Klein (2000), Rokhlin (2008) and Cuchiero et al. (2015). Large financial markets constitute a suitable framework for analysing markets with many assets such as bond markets.

The Arbitrage Pricing Model is a one-period market model containing a countably infinite number of assets. By asymptotic considerations, it makes possible the study of diversification effects in large portfolios. Excluding various kinds of asymptotic arbitrage opportunities leads to qualitative conclusions on the parameters (see Assumption 2.2 below as well as Kabanov \& Kramkov(1998)) which allow for empirical tests and can even be used for asset pricing purposes, see Ross (1976), Huberman (1982), Huang \& Litzenberger (1988).

In the present paper we treat the problem of optimal investment with a criterion of expected utility. Continuing the work initiated in Rásonyi (2016),

*Alfréd Rényi Institute of Mathematics, Hungarian Academy of Sciences, Budapest. Email: rasonyi@renyi.mta.hu 
we seek general conditions under which the existence of optimal portfolios can be asserted for the Arbitrage Pricing Model.

Existence theorems in the context of finitely many assets have been a focus of intensive study, see Kramkov \& Schachermayer (1999), Kabanov \& Stricker (2002), Schachermayer (2001), Biagini \& Frittelli (2005), Owen and Žitković (2009), just to mention a few. Knowing that there is an optimiser brings multiple benefits: it is a reassuring fact per se, since explicit formulas can rarely be found; it helps identifying the conditions on the underlying market and on investor preferences that are necessary for a well-posed problem; finally, it usually constitutes a basis for eventual numerical methods to find the optimiser.

In the context of large financial markets, De Donno et al. (2005) investigated the existence of optimisers in utility maximization for the first time. They worked with the family of "generalized strategies" which, roughly speaking, correspond to the closure (in a suitable topology) of the payoffs of investments into finitely many assets. Thus their optimizer lacks an interpretation in terms of a portfolio in infinitely many assets. Part of the mathematical difficulties related to this problem come from the fact that it is not easy to characterize this closure. The first main contribution of the present paper is to provide reasonable conditions under which a simple characterization is possible, see Lemma 4.3 below. Such results were first obtained in the companion paper Rásonyi (2016), but under restrictive hypotheses which required, in particular, that asset returns take arbitrarily large positive and negative values. In this article we manage to remove this hypothesis on the tails of asset returns at the price of imposing more stringent integrability conditions on them. See Section 3 for further details.

Our second main contribution is establishing that an optimal investment exists, see Theorem 3.5 in Section 3 below. In this way we complement earlier results of Rásonyi (2016). Proofs will be given in Section 4, while Section 5 contains auxiliary results.

\section{Setting}

For $x \in \mathbb{R}$ we denote $x^{+}:=\max \{0, x\}$ and $x^{-}:=\max \{0,-x\}$, the positive/negative parts of $x$. Let $(\Omega, \mathcal{F}, P)$ be a probability space. The expectation of a random variable $X$ with respect to some measure $Q$ on $(\Omega, \mathcal{F})$ is denoted by $E_{Q}[X]$. If $Q=P$ we drop the subscript. $L^{p}$ is the set of $p$-integrable random variables with respect to $P$, for $p \geq 1$. We use $L^{\infty}$ to denote the family of of (essentially) bounded random variables (with respect to $P$ ). When $X \in L^{2}$, we define its variance as

$$
\operatorname{var}(X):=E\left[(X-E X)^{2}\right] .
$$

The symbol $\sim$ denotes equivalence of measures.

We now briefly describe the Arbitrage Pricing Model, concentrating on the mathematical aspects. We assume that the economy in consideration contains assets with returns

$$
\begin{aligned}
R_{0} & :=\quad r ; \quad R_{i}:=\mu_{i}+\bar{\beta}_{i} \varepsilon_{i}, \quad 1 \leq i \leq m \\
R_{i} & :=\mu_{i}+\sum_{j=1}^{m} \beta_{i}^{j} \varepsilon_{j}+\bar{\beta}_{i} \varepsilon_{i}, \quad i>m,
\end{aligned}
$$


where the $\varepsilon_{i}$ are random variables and $\mu_{i}, \beta_{i}, \bar{\beta}_{i}$ are constants. Asset 0 represents a riskless investment with a constant rate of return $r \in \mathbb{R}$. For simplicity, we set $r=0$ from now on.

The random variables $R_{i}, i \geq 1$ represent the profit (or loss) created tomorrow from investing one dollar's worth of asset $i$ today. The random variables $\varepsilon_{i}, i=1, \ldots, m$ serve as factors which influence the return on all the assets $i \geq 1$ while $\varepsilon_{i}, i>m$ are random sources particular to the individual assets $R_{i}, i>m$. We assume that the $\varepsilon_{i}$ are square-integrable, independent random variables satisfying

$$
E\left[\varepsilon_{i}\right]=0, \quad E\left[\varepsilon_{i}^{2}\right]=1, \quad i \geq 1 .
$$

We further assume $\bar{\beta}_{i} \neq 0, i \geq 1$.

Remark 2.1. We deviate from the original definitions of Ross (1976) in two ways. First, in that paper the $\varepsilon_{i}$ are assumed only uncorrelated. It seems, however, that this condition is too weak to obtain interesting results in the present context, see Proposition 4 of Rásonyi (2004). Hence, as in Kabanov \& Kramkov (1998), Rásonyi (2004), we take independent $\varepsilon_{i}$. Second, in Ross (1976) it was not assumed that the factor assets $R_{i}, 1 \leq i \leq m$ are tradeable. This is a rather mild additional assumption we require, just like in Kabanov \& Kramkov (1998), Rásonyi (2004).

A portfolio $\psi$ in the assets $0, \ldots, k$ is an arbitrary sequence $\psi_{i}, 0 \leq i \leq k$ of real numbers satisfying

$$
\sum_{i=0}^{k} \psi_{i}=0 .
$$

This means that we invest $\psi_{i}$ dollars in the respective assets today in such a way that our investments add up to our initial capital 0 (an arbitrary initial capital could be treated similarly, we chose 0 for simplicity again). Such a portfolio will have value

$$
V(\psi):=\sum_{i=0}^{k} \psi_{i} R_{i}
$$

tomorrow.

As $R_{0}=0$, each portfolio is characterized by $\psi_{1}, \ldots, \psi_{k}$. We reparametrize the model by introducing

$$
\begin{aligned}
b_{i} & :=-\frac{\mu_{i}}{\bar{\beta}_{i}}, \quad 1 \leq i \leq m \\
b_{i} & :=-\frac{\mu_{i}}{\bar{\beta}_{i}}+\sum_{j=1}^{m} \frac{\mu_{j} \beta_{i}^{j}}{\bar{\beta}_{j} \bar{\beta}_{i}}, \quad i>m .
\end{aligned}
$$

Asset returns then take the following form:

$$
\begin{aligned}
R_{i} & =\bar{\beta}_{i}\left(\varepsilon_{i}-b_{i}\right), \quad 1 \leq i \leq m \\
R_{i} & =\sum_{j=1}^{m} \beta_{i}^{j}\left(\varepsilon_{j}-b_{j}\right)+\bar{\beta}_{i}\left(\varepsilon_{i}-b_{i}\right), \quad i>m .
\end{aligned}
$$


It is easy to see that the set

$$
J_{1}:=\left\{V(\psi): \psi \text { is a portfolio in assets } R_{0}, \ldots, R_{k}\right\}
$$

coincides with

$$
J_{2}:=\left\{\sum_{i=1}^{k} \phi_{i}\left(\varepsilon_{i}-b_{i}\right): \phi_{1}, \ldots, \phi_{k} \in \mathbb{R}\right\} .
$$

This motivates us to define elementary strategies $\mathcal{E}$ as the set of sequences $\phi=\left\{\phi_{i}, i \geq 1\right\}$ of real numbers such that, for some $k \geq 1, \phi_{i}=0, i>k$. For each $\phi \in \mathcal{E}$, we set

$$
V(\phi):=\sum_{i=1}^{\infty} \phi_{i}\left(\varepsilon_{i}-b_{i}\right) .
$$

This is a slight abuse of notation but it is justified by the equality $J_{1}=J_{2}$. From now on we will use $V(\phi)$ exclusively in the sense (13).

We interpret $\mathcal{E}$ as the set of possible portfolios using only finitely many assets. For $\phi \in \mathcal{E}$, the value tomorrow is given by $V(\phi)$. Define $K:=\{V(\phi): \phi \in \mathcal{E}\}$.

This article is about optimization over the set of portfolios. As $K$ fails to be closed in any reasonable sense, we have no hope for finding an optimiser in it. Hence a natural idea is to enlarge the set of admissible portfolios in the way presented below.

Assumption 2.2. We have

$$
\sum_{i=1}^{\infty} b_{i}^{2}<\infty
$$

As explained in Rásonyi (2004) and in Section 3 of Rásonyi (2016), Assumption 2.2 is equivalent to absence of particular type of asymptotic arbitrage (in the sense of Ross (1976), Huberman (1982)) hence it is a plausible hypothesis on the market parameters.

Let $\ell_{2}:=\left\{\phi_{i}: \sum_{i=1}^{\infty} \phi_{i}^{2}<\infty\right\}$ be the family of square-summable sequences. Recall that $\ell_{2}$ is a Hilbert space with the norm $\|\phi\|_{\ell_{2}}:=\sqrt{\sum_{i=1}^{\infty} \phi_{i}^{2}}$. We set $\mathcal{A}:=\ell_{2}$, this will be the set of strategies over which we will formulate our problem of utility maximization. Under Assumption 2.2, we define $V(\phi):=$ $\sum_{i=1}^{\infty} \phi\left(\varepsilon_{i}-b_{i}\right)$, where the series converges in $L^{2}$.

Now set $K_{1}:=\{V(\phi): \phi \in \mathcal{A}\}$. Taking a supremum over $K_{1}$, it will be possible to find a maximizer inside the same class, under appropriate assumptions, see Theorems 3.2 and 3.5 below.

\section{Existence of optimal strategies}

Let $u: \mathbb{R} \rightarrow \mathbb{R}$ a concave, nondecreasing function. We wish to prove that there is a strategy $\phi^{*} \in \mathcal{A}^{\prime}(u)$ such that

$$
E\left[u\left(V\left(\phi^{*}\right)\right)\right]=\sup _{\phi \in \mathcal{A}^{\prime}(u)} E[u(V(\phi))]
$$

where

$$
\mathcal{A}^{\prime}(u):=\left\{\phi \in \mathcal{A}: E\left[u^{-}(V(\phi))\right]<\infty\right\}
$$

is the set of strategies over which the optimization problem (15) makes sense. 
This is the problem of optimal investment with a risk-averse agent maximizing his/her expected utility from terminal portfolio wealth, see Chapters 2 and 3 of Föllmer \& Schied (2002) for a detailed discussion in the case of finitely many assets.

We recall the results obtained in Rásonyi (2016). The crucial hypothesis in that paper was the following.

Assumption 3.1. For each $x \geq 0$, both

$$
\inf _{i \geq 1} P\left(\varepsilon_{i}>x\right)>0 \text { and } \inf _{i \geq 1} P\left(\varepsilon_{i}<-x\right)>0
$$

hold. Furthermore,

$$
\sup _{i \in \mathbb{N}} E\left[\varepsilon_{i}^{2} 1_{\left\{\left|\varepsilon_{i}\right| \geq N\right\}}\right] \rightarrow 0, N \rightarrow \infty .
$$

We now present Theorem 4.7 of Rásonyi (2016).

Theorem 3.2. Let $u: \mathbb{R} \rightarrow \mathbb{R}$ be concave and non-decreasing, satisfying

$$
u(x) \leq C\left(x^{\alpha}+1\right) \text { for all } x \geq 0,
$$

with some $C>0,0 \leq \alpha<1$. Under Assumptions 2.2 and 3.1, there exists $\phi^{*} \in \mathcal{A}^{\prime}(u)$ such that

$$
E\left[u\left(V\left(\phi^{*}\right)\right)\right]=\sup _{\phi \in \mathcal{A}^{\prime}(u)} E[u(V(\phi))]
$$

In the present article our purpose is to complement Theorem 3.2 by proving the existence of optimizers under alternative conditions. Whereas (18) in Assumption 3.1 is rather mild, (17) is somewhat restrictive: it excludes e.g. the case where all the $\varepsilon_{i}$ are bounded random variables. It would thus be desirable to drop condition (17). In Theorem 3.5 below we manage to do so, at the price of requiring more integrability on the $\varepsilon_{i}$ than (18).

Assumption 3.3. There exists $\gamma>0$ such that

$$
\sup _{i} E\left[e^{\gamma\left|\varepsilon_{i}\right|}\right]<\infty
$$

and, for all $i$,

$$
P\left(\varepsilon_{i}<b_{i}\right), P\left(\varepsilon_{i}>b_{i}\right)>0 .
$$

Remark 3.4. An arbitrage strategy (in the sense of e.g. Föllmer \& Schied $(2002))$ is $\hat{\phi} \in \mathcal{E}$ such that $V(\hat{\phi}) \geq 0$ a.s. and $P(V(\hat{\phi})>0)>0$. We say that the no-arbitrage condition (NA) holds in the given market if there are no arbitrage strategies. (NA) is necessary for the existence of a maximizer for (15) when $u$ is strictly increasing. Indeed, no $\phi^{*}$ can be optimal if $\hat{\phi}$ violates (NA): as $V\left(\phi^{*}+\hat{\phi}\right) \geq V\left(\phi^{*}\right)$ and there is a strict inequality with positive probability, the strategy $\phi^{*}+\hat{\phi}$ outperforms $\phi^{*}$.

Condition (22) is easily seen to imply (NA) in our setting: for any $\phi \in$ $\mathcal{E}$ which is not identically zero, $P\left(\sum_{i=1}^{N} \phi_{i}\left(\varepsilon_{i}-b_{i}\right)<0\right) \geq \prod_{i \in L_{+}(\phi)} P\left(\varepsilon_{i}<\right.$ $\left.b_{i}\right) \prod_{i \in L_{-}(\phi)} P\left(\varepsilon_{i}>b_{i}\right)>0$ since at least one of the two sets $L_{+}(\phi):=\{1 \leq i \leq$ $\left.N: \phi_{i}>0\right\}, L_{-}(\phi):=\left\{1 \leq i \leq N: \phi_{i}<0\right\}$ is non-empty. Conversely, if (22) fails then (NA) is obviously violated. This shows that in our market model (22) is precisely the (NA) condition. 
The main result of this article is stated now.

Theorem 3.5. Let $u: \mathbb{R} \rightarrow \mathbb{R}$ be concave and non-decreasing, satisfying

$$
\begin{aligned}
& u(x) \leq C_{1}\left(x^{\alpha}+1\right) \text { for all } x \geq 0, \\
& u(x) \leq C_{2}\left(-|x|^{\beta}+1\right) \text { for all } x<0
\end{aligned}
$$

with some $C_{1}, C_{2}>0,0 \leq \alpha<1<\beta$. Under Assumptions 2.2 and 3.3, there exists $\phi^{*} \in \mathcal{A}^{\prime}(u)$ such that

$$
E\left[u\left(V\left(\phi^{*}\right)\right)\right]=\sup _{\phi \in \mathcal{A}^{\prime}(u)} E[u(V(\phi))] .
$$

Remark 3.6. Note that neither of Assumptions 3.3, 3.1 implies the other: (17) is stronger than (22) while (18) is weaker than (21). Hence Theorem 3.5 complements Theorem 3.2. Notice also condition (24) in Theorem 3.5 that is absent in Theorem 3.2. As $u$ is concave and non-decreasing, (24) always holds with $\beta=1$. Imposing $\beta>1$ is then only a mild extra requirement.

\section{Proofs}

Condition (21) in Assumption 3.3 implies that the family $\left\{V(\phi): \phi \in \mathcal{E},\|\phi\|_{\ell_{2}} \leq\right.$ $\delta$ \} enjoys a very strong, exponential uniform integrability property, for $\delta>0$ small enough, see the next lemma and the ensuing remark.

Lemma 4.1. Under condition (21), there is $\delta_{0}>0$ such that

$$
\sup _{\phi \in \mathcal{E},\|\phi\|_{\ell_{2}} \leq \delta_{0}} E e^{\left|\sum_{i=1}^{\infty} \phi_{i} \varepsilon_{i}\right|}
$$

is finite.

Proof. For each $i$ and for all $t$ with $|t| \leq \gamma / 2$ there is a random variable $\xi_{i}(t)$ between 0 and $t$ such that

$$
e^{t \varepsilon_{i}}=1+t \varepsilon_{i}+t^{2} \varepsilon_{i}^{2} e^{\xi_{i}(t) \varepsilon_{i}} .
$$

It follows that

$$
E\left[e^{t \varepsilon_{i}}\right] \leq 1+t^{2} E\left[\varepsilon_{i}^{2} e^{\gamma\left|\varepsilon_{i}\right| / 2}\right] .
$$

By elementary properties of the logarithm and by (21),

$$
\left|\ln E\left[e^{t \varepsilon_{i}}\right]\right| \leq c t^{2} E\left[\varepsilon_{i}^{2} e^{\gamma\left|\varepsilon_{i}\right| / 2}\right] \leq C t^{2},
$$

for $t$ small enough, say, for $|t| \leq T$, with some constants $c, C, T>0$ that are independent of $i$. If $\phi_{i}=0$ for $i>N$ then we then have

$$
E\left[e^{\sum_{i=1}^{\infty} \phi_{i} \varepsilon_{i}}\right]=\prod_{i=1}^{N} E\left[e^{\phi_{i} \varepsilon_{i}}\right] \leq e^{C \sum_{i=1}^{N} \phi_{i}^{2}}
$$

provided that $\left|\phi_{i}\right| \leq \min \{T, \gamma / 2\}$ for all $i$. This implies, by $e^{|x|} \leq e^{x}+e^{-x}$,

$$
E\left[e^{\left|\sum_{i=1}^{\infty} \phi_{i} \varepsilon_{i}\right|}\right] \leq 2 e^{C \sum_{i=1}^{N} \phi_{i}^{2}}=2 e^{C\|\phi\|_{\ell_{2}}^{2}},
$$

for all $\phi \in \mathcal{E}$ with $\|\phi\|_{\ell_{2}} \leq \min \{T, \gamma / 2\}$, so one may set $\delta_{0}:=\min \{T, \gamma / 2\}$. 
Remark 4.2. Lemma 4.1 implies that the family $\left\{e^{\left|\sum_{i=0}^{\infty} \phi_{i} \varepsilon_{i}\right|}: \phi \in \mathcal{E},\|\phi \mid\|_{\ell_{2}} \leq\right.$ $\delta\}$ is uniformly integrable for all $\delta<\delta_{0}$, a fortiori, the family $\left\{\left|\sum_{i=0}^{\infty} \phi_{i} \varepsilon_{i}\right|^{2}\right.$ : $\left.\phi \in \mathcal{E},\|\phi\|_{\ell_{2}} \leq 1\right\}$ is also uniformly integrable. It is this latter conclusion that we need in subsequent arguments. We wonder whether this could be deduced from an integrability condition weaker than (21).

We denote by $\bar{K}$ the closure of $K$ with respect to convergence in probability.

Lemma 4.3. Let Assumption 2.2 and (21) in Assumption 3.3 be in force. Then $K_{1}=\bar{K}$ so the set $K_{1}$ is closed in probability.

Proof. We have $\mathcal{E} \subset \mathcal{A}$ and for every $\phi \in \mathcal{A}, V(\phi(n)) \rightarrow V(\phi)$ in $L^{2}$ and hence also in probability, where $\phi(n) \in \mathcal{E}$ is such that $\phi_{i}(n)=\phi_{i}, i \leq n, \phi_{i}(n)=0$, $i>n$. Thus $K$ is dense in $K_{1}$ and it is enough to prove that $K_{1}$ is closed in probability.

Let $\phi(n) \in \mathcal{A}$ such that $V(\phi(n)) \rightarrow X$ a.s. for some random variable $X$. We may and will suppose that $\phi(n) \in \mathcal{E}$ for all $n$. First let us consider the case where $\sup _{n}\|\phi(n)\|_{\ell_{2}}=\infty$. By extracting a subsequence (which we continue to denote by $n$ ) we may and will assume $\|\phi(n)\|_{\ell_{2}} \rightarrow \infty, n \rightarrow \infty$. Define $\tilde{\phi}_{i}(n):=\phi_{i}(n) /\|\phi(n)\|_{\ell_{2}}$ for all $n, i$. Clearly, $\tilde{\phi}(n) \in \mathcal{A}$ with $\|\tilde{\phi}(n)\|_{\ell_{2}}=1$ and

$$
\lim _{n \rightarrow \infty} V(\tilde{\phi}(n))=0 \text { a.s. }
$$

Let $M:=\sqrt{\sum_{i=1}^{\infty} b_{i}^{2}}$. We obviously have $\left|\sum_{i=1}^{\infty} \tilde{\phi}_{i}(n) b_{i}\right| \leq M$ for all $n$.

By Remark 4.2, the family $|V(\tilde{\phi}(n))|^{2}, n \geq 1$ is uniformly integrable which implies that $V(\tilde{\phi}(n)) \rightarrow 0$ in $L^{2}$ as well. But this is absurd since $\operatorname{var}(V(\tilde{\phi}(n)))=$ 1 for all $n \geq 1$. This contradiction shows that necessarily $\sup _{n}\|\phi(n)\|_{\ell_{2}}<\infty$.

Then there is a subsequence which is weakly convergent in $\ell_{2}$ and, by the Banach-Saks theorem applied in $\ell_{2}$, suitable convex combinations

$$
\widehat{\phi}(N):=\frac{1}{N} \sum_{k=1}^{N} \phi_{n_{k}}
$$

with a subsequence $n_{k}$ satisfy

$$
\left\|\widehat{\phi}(N)-\phi^{*}\right\|_{\ell_{2}}^{2}=\sum_{i=1}^{\infty}\left(\widehat{\phi}_{i}(N)-\phi_{i}^{*}\right)^{2} \rightarrow 0, N \rightarrow \infty,
$$

for some $\phi^{*} \in \mathcal{A}=\ell_{2}$. Hence, by orthonormality of the system $\varepsilon_{i}, i \geq 1$ in $L^{2}$,

$$
E\left[\left(V(\widehat{\phi}(N))-V\left(\phi^{*}\right)\right)^{2}\right] \rightarrow 0, \quad N \rightarrow \infty,
$$

so $V(\widehat{\phi}(N)) \rightarrow V\left(\phi^{*}\right)$ in probability as well. As $\widehat{\phi}(N)$ are convex combinations of a subsequence of $\phi(n), V(\widehat{\phi}(N)) \rightarrow X$ in probability. This implies $V\left(\phi^{*}\right)=$ $X$ and the statement of this lemma follows.

Let $\mathcal{M}$ denote the set of $Q \sim P$ such that $E_{Q} R_{i}=0$ for all $i \geq 1$. Elements of $\mathcal{M}$ are the risk-neutral measures for this market model. It is not a priori clear that $\mathcal{M} \neq \emptyset$. 
Remark 4.4. We recall that if $Q \in \mathcal{M}$ is such that $d Q / d P \in L^{2}$ then $E_{Q}[V(\phi)]=0$ for all $\phi \in \mathcal{A}$, see Lemma 3.4 of Rásonyi (2016).

The next ingredient for the proof of Theorem 3.5 is the fact that there are $Q \in \mathcal{M}$ with suitable integrability properties.

Lemma 4.5. Assume that

$$
\sup _{i \geq 1} E\left[\left|\varepsilon_{i}\right|^{3}\right]<\infty
$$

and Assumption 2.2 is in force. Then for all $p \geq 1$ there exists $Q=Q(p) \in \mathcal{M}$ such that $d Q / d P, d P / d Q \in L^{p}$.

Proof. This result essentially follows from Theorem 2 in Rásonyi (2004). We recall the main elements of that argument. Define the function $\psi(x):=1 / 2+$ $1 /\left(1+e^{x}\right), x \in \mathbb{R}$. Using the implicit function theorem, it is shown in the proof of Theorem 2 in Rásonyi (2004) that there exists a sequence $a_{i}, i \geq 1$ and $N^{*} \in \mathbb{N}$ such that for all $N \geq N^{*}$,

$$
\frac{d Q(N)}{d P}:=\prod_{i=N}^{\infty} \frac{\psi\left(a_{i}\left(\varepsilon_{i}-b_{i}\right)\right)}{E \psi\left(a_{i}\left(\varepsilon_{i}-b_{i}\right)\right)}
$$

defines $Q(N) \sim P$ such that $E_{Q(N)}\left[\varepsilon_{i}\right]=b_{i}$ for $i \geq N$. Furthermore, there is a constant $L>0$ such that

$$
\left|a_{i}\right| \leq L\left|b_{i}\right| \text { for } i \geq N^{*} .
$$

It can now be checked by direct calculations that, for each $w \in \mathbb{R}$, the function

$$
(a, b) \rightarrow f_{i}(a, b):=\ln \frac{E\left[\psi^{w}\left(a\left(\varepsilon_{i}-b\right)\right)\right]}{\left(\left[E \psi\left(a\left(\varepsilon_{i}-b\right)\right)\right]\right)^{w}}
$$

is twice continuosly differentiable in a neighbourhood $U$ of $(0,0)$ (which does not depend on $i), f_{i}(0,0)=\partial_{a} f_{i}(0,0)=\partial_{b} f_{i}(0,0)=0$ and the second derivatives are uniformly (in $i$ ) bounded in $U$. This fact was used in Theorem 2 of Rásonyi (2004) for $w=2$ but it holds, in fact, for every $w$.

There is $N^{\prime} \geq N^{*}$ such that $\left(a_{i}, b_{i}\right) \in U$ for $i \geq N^{\prime}$, hence

$$
E\left[\left(\frac{d Q\left(N^{\prime}\right)}{d P}\right)^{w}\right] \leq \prod_{i=N^{\prime}}^{\infty} e^{c \sum_{i=1}^{\infty}\left(a_{i}^{2}+b_{i}^{2}\right)}
$$

for a constant $c>0$. Noting (38) and Assumption 2.2 we conclude that $\left(d Q\left(N^{\prime}\right) / d P\right)^{w} \in L^{1}$. It is clear that the argument also works for two values $w=$ $\pm p$ at the same time and this provides $Q\left(N^{\prime}\right)$ such that $d Q\left(N^{\prime}\right) / d P, d P / d Q\left(N^{\prime}\right) \in$ $L^{p}$.

For $1 \leq i<N^{\prime}$, let $g_{i}:=d W / d P$ where $W$ is given by Corollary 5.2 for the choice $X:=\varepsilon_{i}-b_{i}$. By independence of the $\varepsilon_{i}$, it is clear that

$$
\frac{d Q}{d P}:=\frac{d Q\left(N^{\prime}\right)}{d P} \prod_{i=1}^{N^{\prime}-1} g_{i}
$$

provides a suitable probability $Q$. 
Lemma 4.6. Let $u: \mathbb{R} \rightarrow \mathbb{R}$ be concave and non-decreasing, satisfying

$$
\begin{array}{r}
u(x) \leq C_{1}\left(x^{\alpha}+1\right) \text { for all } x \geq 0, \\
u(x) \leq C_{2}\left(-|x|^{\beta}+1\right) \text { for all } x<0,
\end{array}
$$

with some $C_{1}, C_{2}>0,0 \leq \alpha<1<\beta$. If there exists $Q \in \mathcal{M}$ such that $d Q / d P \in L^{q}, d P / d Q \in L^{r}$, where $q \geq \max \{2, \beta /(\beta-1)\}, r>\alpha /(1-\alpha)$ then there exists $X \in \bar{K}_{1}$ such that

$$
E[u(X)]=\sup _{\phi \in \mathcal{A}^{\prime}(u)} E[u(V(\phi))]
$$

where $\bar{K}_{1}$ denotes the closure of $K_{1}$ for convergence in probability.

Proof. The arguments in this proof are inspired by those of Theorem 4.7 in Rásonyi (2016). Denote $Y:=V(\phi)$ for some $\phi \in \mathcal{A}^{\prime}(u)$. By Hölder's inequality, Remark 4.4 and (42),

$$
\begin{aligned}
E\left[u\left(Y^{+}\right)\right] & \leq C_{1}\left(E\left[\left(Y^{+}\right)^{\alpha}\right]+1\right) \leq C_{1}\left(C^{\prime}\left(E_{Q}\left[Y^{+}\right]\right)^{\alpha}+1\right)= \\
C_{1}\left(C^{\prime}\left(E_{Q}\left[Y^{-}\right]\right)^{\alpha}+1\right) & \leq C_{1}\left(C^{\prime} C^{\prime \prime}\left(E\left[\left(Y^{-}\right)^{\beta}\right]\right)^{\alpha / \beta}+1\right) \\
& \leq C_{1}\left(C^{\prime} C^{\prime \prime}\left(-\left(1 / C_{2}\right) E\left[u\left(-Y^{-}\right)\right]+1\right)^{\alpha / \beta}+1\right)(45)
\end{aligned}
$$

with $C^{\prime}:=\left(E\left[(d P / d Q)^{\alpha /(1-\alpha)}\right]\right)^{1-\alpha}, C^{\prime \prime}:=\left(E\left[(d Q / d P)^{\beta /(\beta-1)}\right]\right)^{\alpha(\beta-1) / \beta}$. Let $\phi_{n} \in \mathcal{A}^{\prime}(u)$ be such that $E\left[u\left(V\left(\phi_{n}\right)\right)\right] \rightarrow \sup _{\phi \in \mathcal{A}^{\prime}(u)} E[u(V(\phi))], n \rightarrow \infty$. If we had $\sup _{n} E_{Q}\left[\left|V\left(\phi_{n}\right)\right|\right]=\infty$ then (along a subsequence), $E\left[u\left(-V^{-}\left(\phi_{n}\right)\right)\right] \rightarrow$ $-\infty, n \rightarrow \infty$, by (45). Using the fact that for a non-increasing function $u$ one has

$$
u(x) \leq u\left(x^{+}\right)+u\left(-x^{-}\right)+|u(0)|,
$$

it follows that

$$
E\left[u\left(V\left(\phi_{n}\right)\right)\right] \leq E\left[u\left(V^{+}\left(\phi_{n}\right)\right)\right]+E\left[u\left(-V^{-}\left(\phi_{n}\right)\right)\right]+|u(0)| \rightarrow-\infty,
$$

by (45) and by $\alpha / \beta<1$, which is absurd. Hence $\sup _{n} E_{Q}\left[\left|V\left(\phi_{n}\right)\right|\right]$ must be finite and Komlós's theorem (see Komlós (1967)) implies that, for some convex combinations of a subsequence,

$$
\widehat{\phi}_{N}=\frac{1}{N} \sum_{k=1}^{N} \phi_{n_{k}}
$$

one has $V\left(\widehat{\phi}_{N}\right) \rightarrow X$ a.s., $N \rightarrow \infty$. By convexity of $K_{1}$, clearly $X \in \bar{K}_{1}$. We claim that $X$ satisfies (44).

Indeed, choose $\theta>\alpha$ such that $\theta /(1-\theta)=r$. We get by Hölder's inequality,

$$
E\left[\left(V^{+}\left(\widehat{\phi}_{N}\right)\right)^{\theta}\right] \leq C^{\prime \prime \prime}\left(E_{Q}\left[V^{+}\left(\widehat{\phi}_{N}\right)\right]\right)^{\theta} \leq C^{\prime \prime \prime}\left(\sup _{n} E_{Q}\left[\left|V\left(\phi_{n}\right)\right|\right]\right)^{\theta}
$$

with constant $C^{\prime \prime \prime}:=\left(E\left[(d P / d Q)^{\theta /(1-\theta)}\right]\right)^{1-\theta}$, for all $n$, recalling that the $\widehat{\phi}_{N}$ are convex combinations of the $\phi_{n}$. As the latter expression has a finite supremum in $n$ by our arguments above, we get by (42) that the family $u\left(V^{+}\left(\widehat{\phi}_{N}\right)\right)$, 
$N \in \mathbb{N}$ is uniformly integrable. This, combined with Fatou's lemma and the convexity of $u$, implies

$$
E[u(X)] \geq \limsup _{N \rightarrow \infty} E\left[u\left(V\left(\widehat{\phi}_{N}\right)\right)\right] \geq \limsup _{n \rightarrow \infty} E\left[u\left(V\left(\phi_{n}\right)\right)\right],
$$

and the proof is finished.

Proof of Theorem 3.5. Notice that Assumption 3.3 implies (36). Choose $p$ with $p>\max \{2, \beta /(\beta-1), \alpha /(1-\alpha)\}$ and invoke Lemma 4.5. From Lemma 4.6 we get a maximizer $X \in \bar{K}_{1}$ and, by Lemma 4.3, $X=V\left(\phi^{*}\right)$ for some $\phi^{*} \in \mathcal{A}$. Since $0 \in \mathcal{A}^{\prime}(u), E\left[u\left(V\left(\phi^{*}\right)\right)\right] \geq E[u(0)]>-\infty$ so $\phi^{*} \in \mathcal{A}^{\prime}(u)$ as well and the proof is finished.

Remark 4.7. It is clear from the above proofs that $E\left[u\left(X^{+}\right)\right]<\infty$ hence

$$
E\left[u\left(V\left(\phi^{*}\right)\right)\right]=\sup _{\phi \in \mathcal{A}^{\prime}(u)} E[u(V(\phi))]<\infty .
$$

\section{Appendix}

Here we briefly treat utility maximization for the case of one asset, which is rather elementary but indispensable for the proof of Theorem 3.5. The arguments below are standard, see e.g. Davis (1997), Föllmer \& Schied (2002), but we could not find a reference that would cover the setting we need.

Let $X$ be a real-valued random variable with $P(X>0), P(X<0)>0$ and $E|X|<\infty$. Let furthermore $u$ be concave and nondecreasing, $u(x)=c x, x<0$, $u(x) \leq C\left(x^{\alpha}+1\right)$ for $x \geq 0$ with some $c, C>0,0 \leq \alpha<1$.

Proposition 5.1. There exists $\phi^{*} \in \mathbb{R}$ such that

$$
\sup _{\phi \in \mathbb{R}} E[u(\phi X)]=E\left[u\left(\phi^{*} X\right)\right] .
$$

Proof. Let $\phi>0$ first. Then $E[u(\phi X)] \leq C\left(\phi^{\alpha} E\left[\left(X^{+}\right)^{\alpha}\right]+1\right)-\phi c E\left[X^{-}\right]$ and this tends to $-\infty$ as $\phi \rightarrow \infty$ since $E\left[X^{-}\right]>0$. A similar argument shows that $E[u(\phi X)] \rightarrow-\infty$ as $\phi \rightarrow-\infty$. The function $\phi \rightarrow E[u(\phi)]$ is continuous. To see this, let $\phi_{n} \rightarrow \phi$. Clearly, for $D:=\sup _{n}\left|\phi_{n}\right|+1$ we have $\left|u\left(\phi_{n} X\right)\right| \leq \max \{c, C\}[(D|X|+1)+1]$ for all $n$, using the inequality $x^{\alpha} \leq x+1$. Lebesgue's theorem implies $E\left[u\left(\phi_{n} X\right)\right] \rightarrow E[u(\phi X)]$. Now the statement is clear.

Corollary 5.2. For each $p \geq 1$ there is $W=W(p) \sim P$ with $E_{W}[X]=0$ such that $d W / d P \in L^{\infty}$ and $d P / d W \in L^{p}$.

Proof. Define $u(x)=\alpha x, x \leq 0$ and $u(x)=(x+1)^{\alpha}-1$ for $x>0$ with some $0<\alpha<1$. Clearly, $u$ is continuously differentiable. We claim that $\phi \rightarrow E[u(\phi X)]$ is also continuously differentiable. It suffices to show that the set of random variables $u^{\prime}(\phi X) X, \phi \in \mathbb{R}$ is dominated by a random variable of finite expectation, which is clear as $\left|u^{\prime}(\phi X) X\right| \leq \alpha|X|$. By Proposition 5.1, the function $\phi \rightarrow E[u(\phi X)]$ attains its maximum at $\phi^{*}$, so $E\left[u^{\prime}\left(\phi^{*} X\right) X\right]=0$ which implies $E_{W}[X]=0$ for the probability $W \sim P$ defined by

$$
\frac{d W}{d P}:=\frac{u^{\prime}\left(\phi^{*} X\right)}{E\left[u^{\prime}\left(\phi^{*} X\right)\right]} .
$$


As $u^{\prime}$ is bounded, $d W / d P \in L^{\infty}$. We also have that $d P / d W$ is smaller than constant times $\left(\left|\phi^{*} X\right|+1\right)^{1-\alpha}$. Since $X$ has a finite expectation, the latter expression is in $L^{p}$ for an arbitrarily large $p$ provided that $\alpha$ is chosen close enough to 1 .

Acknowledgments. I thank the referee for useful comments. This work was supported by the Hungarian Academy of Sciences ["Lendület" Grant LP2015$6]$.

\section{References}

[1] S. Biagini \& M. Frittelli (2005) Utility maximization in incomplete markets for unbounded processes, Finance Stoch. 9, 493-517.

[2] C. Cuchiero, I. Klein \& J. Teichmann (2015) A new perspective on the fundamental theorem of asset pricing for large financial markets, Teoriya Veroyatnostei i ee Primeneniya, 60, 660-686. Forthcoming in Theory of Probability and its Applications, 2016.arXiv:1412.7562

[3] M.H.A. Davis (1997) Option pricing in incomplete markets, In: Mathematics of derivative securities (M. A. H. Dempster and S. R. Pliska, ed.), 216-226, Cambridge University Press.

[4] M. De Donno, P. Guasoni \& M. Pratelli (2005) Superreplication and utility maximization in large financial markets. Stochastic Process. Appl. 115, 2006-2022.

[5] H. Föllmer \& A. Schied (2002) Stochastic finance: an introduction in discrete time. Walter de Gruyter \& Co., Berlin.

[6] Ch.-F. Huang \& R. H. Litzenberger (1988) Foundations for financial economics. Elsevier.

[7] G. Huberman (1982) A simple approach to arbitrage pricing theory, $J$. Econom. Theory 28, 289-297.

[8] Yu. M. Kabanov \& D. O. Kramkov (1994) Large financial markets: asymptotic arbitrage and contiguity, Theory Probab. Appl. 39, 182-187.

[9] Yu. M. Kabanov \& D. O. Kramkov (1998) Asymptotic arbitrage in large financial markets, Finance Stoch. 2, 143-172.

[10] Yu. M. Kabanov \& Ch. Stricker (2002) On the optimal portfolio for the exponential utility maximization: remarks to the six-author paper, Math. Finance 12, 125-134.

[11] I. Klein (2000) A fundamental theorem of asset pricing for large financial markets, Math. Finance, 10, 443-458.

[12] I. Klein \& W. Schachermayer (1996a) Asymptotic arbitrage in noncomplete large financial markets, Theory Probab. Appl. 41, 780-788. 
[13] I. Klein \& W. Schachermayer (1996b) A quantitative and a dual version of the Halmos-Savage theorem with applications to mathematical finance, Ann. Probab. 24, 867-881.

[14] J. Komlós (1967) A generalization of a problem of Steinhaus, Acta Math. Acad. Sci. Hungar. 18, 217-229.

[15] D. O. Kramkov \& W. Schachermayer (1999) The asymptotic elasticity of utility functions and optimal investment in incomplete markets, Ann. Appl. Probab. 9, 904-950.

[16] M. P. Owen \& G. Žitković (2009) Optimal investment with an unbounded random endowment and utility-based pricing, Math. Finance 19, 129-159.

[17] M. Rásonyi (2004) Arbitrage pricing theory and risk-neutral measures, Decis. Econ. Finance 27, 109-123.

[18] M. Rásonyi (2016) Maximizing expected utility in the Arbitrage Pricing Model, Submitted, arXiv:1508.07761v2

[19] S. A. Ross. (1976) The arbitrage theory of capital asset pricing, J. Econom. Theory 13, 341-360.

[20] D. B. Rokhlin (2008) Asymptotic arbitrage and numéraire portfolios in large financial markets, Finance Stoch. 12, 173-194.

[21] W. Schachermayer (2001) Optimal investment in incomplete markets when wealth may become negative, Ann. Appl. Probab. 11, 694-734. 